\title{
Holomorphic factorization of matrices of polynomials
}

\author{
John P. D'Angelo \\ Dept. of Mathematics \\ University of Illinois \\ Urbana IL 61801 \\ USA
}

\section{Introduction}

This paper considers some work done by the author and Catlin [CD1,CD2,CD3] concerning positivity conditions for bihomogeneous polynomials and metrics on bundles over certain complex manifolds. It presents a simpler proof of a special case of the main result in [CD3], providing also a self-contained proof of a generalization of the main result from [CD1]. Some new examples and applications appear here as well. The idea is to use the Bergman kernel function and some operator theory to prove purely algebraic theorems about matrices of polynomials.

The main idea arises from generalizing a classical factorization question. See [Dj] and $[R R]$ for many aspects of factorization of non-negative matrices and operators on Hilbert spaces. Consider a real-analytic matrix-valued function $F(z, \bar{z})$ that is positive semi-definite at each point. Is there a holomorphic matrix-valued function $A(z)$ such that $F(z, \bar{z})=A(z)^{*} A(z)$ ? Here $A^{*}$ denotes the conjugate transpose of $A$. In general the answer is no, even when $F$ is a scalar, positive-definite, and its entries are bihomogeneous polynomials. Because such factorizations have many applications, we allow ourselves a generalization; we can first multiply $F$ by powers of a scalar function $R$, and ask whether we can factor $R^{d} F$ for sufficiently large $d$. This is a natural thing to do when one studies proper holomorphic mappings between balls in different dimensions, and one chooses $R$ to be the squared Euclidean norm. See [CD1] for applications. This multiplication also admits an interpretation in terms of metrics on tensor products of Hermitian line bundles.

We write $\langle\zeta, w\rangle$ for the Euclidean Hermitian inner product of $\zeta$ and $w$ on any finitedimensional complex Euclidean space, and $\|\zeta\|^{2}$ for the squared norm. Later we will use subscripts to denote $L^{2}$ norms. We write $\mathbf{V}(A)$ to denote the variety defined by the simultaneous vanishing of the component functions of a holomorphic mapping $A$.

A bihomogeneous polynomial on $\mathbf{C}^{\mathbf{n}}$ is a polynomial function $f: \mathbf{C}^{\mathbf{n}} \times \mathbf{C}^{\mathbf{n}} \rightarrow \mathbf{C}$ that is homogeneous of the same degree $m$ in each set of variables. We will be considering $f(z, \bar{w})$, which is conjugate-analytic in the second set of variables. The polynomial defined by $f(z, \bar{z})$ is real-valued if and only if $f(z, \bar{w})=\overline{f(w, \bar{z})}$; we call such an $f$ a bihomogeneous real-valued polynomial on $\mathbf{C}^{\mathbf{n}}$ of degree $2 m$.

Suppose that $f$ is a bihomogeneous real-valued polynomial of degree $2 m$. By elementary linear algebra, it is possible to find holomorphic polynomial mappings $A$ and $B$, with finitely many components, that are homogeneous of degree $m$, and such that

$$
f(z, \bar{w})=\langle A(z), A(w)\rangle-\langle B(z), B(w)\rangle
$$

It follows from (1) that

$$
f(z, \bar{z})=\|A(z)\|^{2}-\|B(z)\|^{2}
$$


Suppose in addition that $f(z, \bar{z}) \geq 0$. We investigate the following questions. Can we choose $B=0$ in (2), and if we cannot, can we do this for $R^{d} f$, where $R$ is an appropriate multiplier and $d$ is sufficiently large? Suppose more generally that $F(z, \bar{z})$ is a matrix of bihomogeneous polynomials each of degree $2 m$, and that is positive semi-definite at each $z \neq 0$. Can we factor $\|z\|^{2 d} F(z, \bar{z})$ for sufficiently large $d$ ?

The following theorem gives a decisive answer in the positive-definite case.

Theorem 1. [Catlin-D'Angelo]. Suppose that $f$ is a bihomogeneous real-valued polynomial on $\mathbf{C}^{\mathbf{n}}$ of degree $2 m$. Then $f$ is positive away from the origin if and only there is an integer $d$ and a holomorphic homogeneous polynomial mapping $A$, whose components span the space of homogeneous polynomials of degree $m+d$, such that

$$
\|z\|^{2 d} f(z, \bar{z})=\|A(z)\|^{2}
$$

Suppose that $F(z, \bar{z})$ is an $r$ by $r$ matrix whose entries are bihomogeneous polynomials of degree $2 m$. Then $F(z, \bar{z})$ is positive-definite at each point $z \neq 0$ if and only if there is an integer $d$ and a holomorphic homogeneous polynomial matrix $A$, whose row vectors span the space of $r$-tuples of homogeneous polynomials of degree $m+d$, such that

$$
\|z\|^{2 d} F(z, \bar{z})=A(z)^{*} A(z) .
$$

Note that (3) is the scalar version of (4). The scalar statement about $f$ was proved in [CD1]. The matrix version is a special case of a general result from [CD3] about Hermitian metrics on bundles over certain complex manifolds. Because it is a special case, some steps in the proof simplify; its intrinsic interest justifies giving the simpler proof here. In the scalar statement, one can replace the condition that the components of $A$ span, by the condition that $\mathbf{V}(A)=\{0\}$; the exponent required may be smaller. The proof reveals that the stronger condition on $A$ is the natural one.

When $F(z, \bar{z})=A(z)^{*} A(z)$, necessarily $F(z, \bar{z})$ is positive semi-definite at each point. A general result such as Theorem 1 cannot hold in the positive semi-definite case, for the following simple reason. Suppose $r=1$ for simplicity. If the zero set of $f$ is not an analytic variety, then there is no hope to write $\|z\|^{2 d} f(z, \bar{z})=\|A(z)\|^{2}$, because the zero set of the right side is an analytic variety. A simple example where the zero set of a bihomogeneous polynomial fails to be an analytic variety is given by (5).

$$
f(z, \bar{z})=\left(\left|z_{1}\right|^{2}-\left|z_{2}\right|^{2}\right)^{2}
$$

Some results hold under specific hypotheses in the semi-definite case, but most of this paper considers only the positive-definite case.

We give a complete proof of Theorem 1, relying on the Bergman kernel function for the unit ball. In Theorem 2 we give a simple application to elliptic PDE. In Theorem 3 we reinterpret Theorem 1 in terms of the universal bundle over complex projective space. We also provide some illuminating examples along the way. We close the paper with some brief remarks about factorization theorems proved in the 1970s. 


\section{Holomorphic factorability}

Suppose that we are given an $r$ by $r$ matrix $F(z, \bar{w})$ whose entries $F_{i j}(z, \bar{w})$ are bihomogeneous polynomials of degree $2 m$ on $\mathbf{C}^{\mathbf{n}}$. Let $N=N(n, m)$ denote the dimension of the vector space of homogeneous polynomials of degree $m$ in $n$ variables. We write $V_{d}$ for the vector space of $r$-tuples of homogeneous polynomials of degree $d$ on $\mathbf{C}^{\mathbf{n}}$.

Definition 1. The $r$ by $r$ matrix of bihomogeneous polynomials $F(z, \bar{w})$ of degree $2 m$ is called holomorphically factorable if there is an integer $s$ and a matrix $\left(E_{j k}(z)\right)$, for $j=1, \ldots, r$ and $k=1, \ldots, s$, of homogeneous polynomials of degree $m$ such that (6) holds.

$$
F_{i j}(z, \bar{w})=\left\langle E_{j}(z), E_{i}(w)\right\rangle=\sum_{k=1}^{s} E_{j k}(z) \overline{E_{i k}(w)}
$$

Here $E_{j}(z)=\left(E_{j 1}(z), \ldots, E_{j s}(z)\right)$.

Let $A$ be the transpose of $E$; we observe immediately that Definition 1 implies that

$$
F(z, \bar{w})=A(w)^{*} A(z) .
$$

Definition 2. The matrix of bihomogeneous polynomials $F(z, \bar{w})$ of degree $2 m$ is called strictly holomorphically factorable if it is holomorphically factorable, and in addition the $s$ column vectors of $\left(E_{j k}(z)\right)$ are a basis for $V_{d}$.

Note that the notion of strict holomorphic factorability requires that we choose $s=N$. The first concept allows us to write $(7)$, forcing $F(z, \bar{z})$ to be positive semi-definite. The second concept ensures that $F(z, \bar{z})$ is positive-definite, but it is an even stronger condition. We give a simple example when $r=1$ to illustrate the difference.

Example. Let $F(z, \bar{w})$ be the one-by-one matrix given by $z_{1}^{2} \bar{w}_{1}^{2}+z_{2}^{2} \bar{w}_{2}{ }^{2}$. Here Definition 1 holds with $E_{1}(z)=\left(z_{1}^{2}, z_{2}^{2}\right)$. Note that $F(z, \bar{z})$ is positive-definite away from the origin, but the absence of the $z_{1} z_{2}$ cross-term means that Definition 2 fails.

\section{The link to operator theory}

We will prove Theorem 1 by using some facts about compact operators on a Hilbert space. Because we consider (matrices of) bihomogeneous polynomials and use the Euclidean norm as a multiplier, it suffices to consider the Hilbert space $\mathcal{H}$ of $r$-tuples of $L^{2}$ functions on the unit ball $B$ in complex Euclidean space $\mathbf{C}^{\mathbf{n}}$. We let $\mathcal{A}$ denote the closed subspace of $r$-tuples of holomorphic functions in $L^{2}(B)$. We write $P$ for the Bergman projection from $\mathcal{H}$ to $\mathcal{A}$; it is the usual Bergman projection acting on each component.

There is an orthogonal decomposition of $\mathcal{A}$ into the finite-dimensional subspaces $V_{d}$. The following result provides the crucial link between matrices whose entries are bihomogeneous polynomials and operator theory on $\mathcal{A}$.

Proposition. Let $F(z, \bar{z})=\left(F_{i j}(z, \bar{z})\right)$ be an $r$ by $r$ matrix of bihomogeneous polynomials of degree $2 d$ on $\mathbf{C}^{\mathbf{n}}$. The following are equivalent:

1. The matrix $F$ is strictly holomorphically factorable. Thus there is an $s$ by $r$ matrix $E(z)$ of holomorphic homogeneous polynomials whose column vectors give a basis for $V_{d}$ for each $z \neq 0$. Furthermore, with $A$ the transpose of $E$, (8) holds. 


$$
F(z, \bar{w})=A(w)^{*} A(z)
$$

2. Consider the integral operator $T: V_{d} \rightarrow V_{d}$ defined by

$$
T h(z)_{j}=\int_{B} \sum_{i} F_{i j}(z, \bar{w}) h_{i}(w) d V(w)
$$

Then $T$ is positive on $V_{d}$. That is, there is a positive constant $c$ so that for $h \in V_{d}$,

$$
\langle T h, h\rangle_{\mathcal{H}} \geq c\|h\|_{\mathcal{H}}^{2}
$$

3. Write

$$
F_{i j}(z, \bar{w})=\sum F_{i j \alpha \beta} z^{\alpha} \bar{w}^{\beta}
$$

for constants $F_{i j \alpha \beta}$. Then the matrix $\left(F_{i j \alpha \beta}\right)$ is positive-definite on $\mathbf{C}^{\mathbf{N r}}=\mathbf{C}^{\mathbf{r}} \otimes \mathbf{C}^{\mathbf{N}}$; that is, there is a positive constant $c^{\prime}$ so that

$$
\sum F_{i j \alpha \beta} t_{i} \bar{t}_{j} s_{\alpha} \bar{s}_{\beta} \geq c^{\prime} \sum\left|t_{i} s_{\alpha}\right|^{2}=c^{\prime} \sum\left|t_{i}\right|^{2} \sum\left|s_{\alpha}\right|^{2}
$$

Proof. First we show that 1 implies 2. Given $h \in V_{d} \subset \mathcal{A}$, we write $h=\left(h_{1}, \ldots, h_{r}\right)$. We assume that (6) holds and compute the left side of (10), to obtain

$$
\begin{gathered}
\langle T h, h\rangle_{\mathcal{H}}=\iint \sum_{k=1}^{s} \sum_{i, j=1}^{r} \overline{E_{i k}(w)} E_{j k}(z) h_{i}(w) \overline{h_{j}(z)} d V(w) d V(z) \\
=\sum_{k=1}^{s}\left|\int \sum_{j} E_{j k}(z) \overline{h_{j}(z)} d V(z)\right|^{2}=\sum_{k=1}^{s}\left|\left\langle A_{k}, h\right\rangle_{\mathcal{H}}\right|^{2}
\end{gathered}
$$

The condition of strict holomorphic factorability guarantees that the vectors $A_{k}$, the column vectors of $E$, form a basis for $V_{d}$. The last expression in (13) is therefore $\geq c\|h\|_{\mathcal{H}}^{2}$. This proves that 1 implies 2.

Next we show that 3 implies 1 . Recall that $N$ is the dimension of the space of homogeneous polynomials of degree $d$ in $n$ variables, and that $\left(F_{i j}\right)$ is an $r$ by $r$ matrix. If (12) holds, there is a basis $\left\{E_{i \beta}\right\}$ of $\mathbf{C}^{\mathbf{N r}}$ so that $F_{i j \alpha \beta}=\left\langle E_{j \alpha}, E_{i \beta}\right\rangle$. Plug this in (11) to obtain

$$
F_{i j}(z, \bar{w})=\sum F_{i j \alpha \beta} z^{\alpha} \bar{w}^{\beta}=\sum\left\langle E_{j \alpha}, E_{i \beta}\right\rangle z^{\alpha} \bar{w}^{\beta}
$$

Now define $A_{j}(z)$ by $A_{j}(z)=\sum E_{j \alpha} z^{\alpha}$. We see that

$$
F_{i j}(z, \bar{w})=\left\langle A_{j}(z), A_{i}(w)\right\rangle
$$

and hence that 1 holds.

It remains to prove that 2 implies 3 . We write $h_{i}(z)=\sum H_{i \alpha} z^{\alpha}$, and we plug this into $\langle T h, h\rangle_{\mathcal{H}}$. Recall that distinct monomials are orthogonal, so we may write 


$$
\langle T h, h\rangle_{\mathcal{H}}=\sum \iint F_{i j \alpha \beta} z^{\alpha} \bar{w}^{\beta} H_{i \mu} w^{\mu} \bar{H}_{j \nu} \bar{z}^{\nu} d V(w) d V(z)=\sum F_{i j \alpha \beta} H_{i \beta} \bar{H}_{j \alpha} p_{\alpha} p_{\beta}
$$

where the positive numbers $p_{\alpha}$ are equal to $\left\|z^{\alpha}\right\|_{L^{2}}^{2}$.

On the other hand, we have $\|h\|_{\mathcal{H}}^{2}=\sum\left|H_{i \alpha}\right|^{2} p_{\alpha}$ by a similar calculation. Thus (10) implies that there is a positive constant $c$ so that

$$
\sum F_{i j \alpha \beta} p_{\alpha} p_{\beta} H_{i \beta} \bar{H}_{j \alpha} \geq c \sum\left|H_{i \alpha}\right|^{2} p_{\alpha}
$$

Since the $p_{\beta}$ are positive numbers, (17) implies that the matrix with entries $F_{i j \alpha \beta}$ is also positive-definite, with a different constant $c^{\prime}$. This gives 3 .

\section{Proof of Theorem 1}

Suppose that the entries of $F$ are bihomogeneous polynomials of degree $2 m$. Let $Q_{d}$ be the operator on $V_{m+d}$ whose kernel is given by $\langle z, w\rangle^{d} F(z, \bar{w})$. In order to prove Theorem 1, Proposition 1 implies that we must find an integer $d$ so that $Q_{d}$ is positive-definite on $V_{m+d}$. We observe immediately, that if this holds for some $d$, then it holds for all larger integers. See [CD1], whose title suggests this stabilization process. Furthermore, the operators $Q_{d}$ are zero except on $V_{m+d}$. This suggests considering their sum, weighted by positive constants, on the whole space $\mathcal{A}$.

If we choose the positive constants $C_{d}$ appropriately, then

$$
\sum C_{d}\langle z, w\rangle^{d}=\frac{n !}{\pi^{n}} \frac{1}{(1-\langle z, w\rangle)^{n+1}}=B(z, w)
$$

Here $B(z, w)$ is the Bergman kernel function for the unit ball $B$ in complex Euclidean space $\mathbf{C}^{\mathbf{n}}$. The crucial property of the Bergman kernel function is that it is the integral kernel of the Bergman projection mapping $L^{2}(B)$ to its closed subspace $A^{2}(B)$ of holomorphic functions. The kernel function satisfies

$$
B(z, w)=\sum_{\alpha} \phi_{\alpha}(z) \overline{\phi_{\alpha}(w)}
$$

where the collection $\left\{\phi_{\alpha}\right\}$ is any complete orthonormal set for the Hilbert space $A^{2}(B)$. For the unit ball, one can choose $\phi_{\alpha}=c_{\alpha} z^{\alpha}$, where $c_{\alpha}$ is a normalizing constant, and $z^{\alpha}$ denotes the indicated monomial.

Recall that $\mathcal{H}$ denotes the Cartesian product of $r$ copies of $L^{2}(B)$. Let $P: \mathcal{H} \rightarrow \mathcal{A}$ denote the Bergman projection, acting componentwise. Motivated by (18), we let $Q$ denote the integral operator on $\mathcal{H}$ whose kernel is given by $B(z, w) F(z, \bar{w})$. For a scalar function $\psi$, we let $M_{\psi}$ denote the operator on $\mathcal{H}$ given by multiplication by $\psi$. Also $M_{F}$ denotes matrix multiplication by $F$. There is no integration involved in these operators. Choose a smooth non-negative function $\phi$ of compact support that is positive near the origin.

We may write 


$$
Q=\left(M_{F} P+P M_{\phi}\right)+\left(Q-M_{F} P\right)-P M_{\phi}=T_{1}+T_{2}+T_{3}
$$

We claim that $T_{1}$ is positive, and that $T_{2}$ and $T_{3}$ are compact. This will show that $Q=S+K$, where $S$ is positive on $\mathcal{A}$ and $K$ is compact.

Lemma 1. $T_{1}$ is positive on all of $\mathcal{A}$, and $T_{3}$ is compact on $\mathcal{H}$.

Proof. The second statement is immediate, because the integral kernel is smooth everywhere on the ball. The first statement follows because $P$ is a self-adjoint projection. To see this, let $h \in \mathcal{A}$.

$$
\left\langle T_{1} h, h\right\rangle_{\mathcal{H}}=\left\langle M_{F} P h+P M_{\phi} h, h\right\rangle_{\mathcal{H}}=\left\langle M_{F} h, h\right\rangle_{\mathcal{H}}+\left\langle M_{\phi} h, h\right\rangle_{\mathcal{H}}=\left\langle M_{F+\phi} h, h\right\rangle_{\mathcal{H}}
$$

Since the multiplier $F+\phi$ is strictly positive-definite at all points, the last expression in (20) is at least $C \mid\|h\|_{\mathcal{H}}^{2}$, and the result follows.

Lemma 2. $T_{2}$ is compact.

Proof. This follows from Theorem 1 in [CD2], but is elementary in this case, because of the explicit nature of the Bergman kernel. The kernel of $Q-M_{F} P$ is

$$
\frac{n !}{\pi^{n}} \frac{(F(z, \bar{w})-F(z, \bar{z}))}{(1-\langle z, w\rangle)^{n+1}}
$$

The numerator (a matrix of polynomials) vanishes on the boundary diagonal, where the only singularities of the denominator occur. One can use Young's inequality to verify that $T_{2}$ is compact.

We summarize what we have proved so far. The operator $Q$ on $\mathcal{H}$ has kernel given by $B(z, w) F(z, \bar{w})$. By Lemmas 1 and 2, we have written $Q=S+K$, where $S$ is positive on $\mathcal{A}$ and $K$ is compact.

The operator $Q$ vanishes off $\mathcal{A}$, and we have $\mathcal{A}=\oplus V_{j}$. Write $Q_{d}$ for the restriction of $Q$ to $V_{m+d}$. If we show that $Q_{d}$ is positive on $V_{m+d}$ for sufficiently large $d$, then an application of Proposition 1 completes the proof of Theorem 1.

Since $S$ is positive, there is $c>0$ so that $\langle S h, h\rangle_{\mathcal{H}} \geq c\|h\|_{\mathcal{H}}^{2}$. Since $K$ is compact, there is a finite rank operator $L$ such that the operator norm $\||| K-L \mid\|<\frac{c}{3}$. See $[\mathrm{R}]$. Write $Q=S+L+(K-L)$ so that

$$
\langle Q h, h\rangle_{\mathcal{H}}=\langle S h, h\rangle_{\mathcal{H}}+\langle L h, h\rangle_{\mathcal{H}}+\langle(K-L) h, h\rangle_{\mathcal{H}}
$$

Using the lower bound on $S$, and because $\left|\langle(K-L) h, h\rangle_{\mathcal{H}}\right| \leq\left.\frac{c}{3}|| h\right|_{\mathcal{H}} ^{2}$, we can write

$$
\langle Q h, h\rangle_{\mathcal{H}} \geq c|| h\left\|_{\mathcal{H}}^{2}-\frac{c}{3}\right\| h\left\|_{\mathcal{H}}^{2}-\left|\langle L h, h\rangle_{\mathcal{H}}\right| \geq \frac{2 c}{3}\right\| h \|_{\mathcal{H}}^{2}-\left|\langle L h, h\rangle_{\mathcal{H}}\right|
$$

Because $L$ is finite rank, we can choose $d_{0}$ sufficiently large such that, for $d \geq d_{0}$, the restriction of $L$ to $V_{m+d}$ satisfies $\left|\langle L h, h\rangle_{\mathcal{H}}\right| \leq \frac{c}{3}|| h \|_{\mathcal{H}}^{2}$ also. Combining this with (22) implies that the restriction of $Q$ to $V_{m+d}$ is positive. By Proposition 1, its kernel $\langle z, w\rangle^{d} F(z, \bar{w})$ can be written $A(w)^{*} A(z)$, completing the proof of Theorem 1 . 


\section{Examples and Applications.}

The integer $d$ in Theorem 1 can be arbitarily large even when $F$ has fixed degree. The example $f_{c}(z, \bar{z})=\left|z_{1}\right|^{4}+c\left|z_{1} z_{2}\right|^{2}+\left|z_{2}\right|^{4}$ is positive away from the origin for $c>-2$. By Theorem 1, for each $c$ with $c>-2$, there is a minimum $d_{c}$ for which (3) holds. It is elementary to show that $d_{c} \rightarrow \infty$ as $c \rightarrow-2$. See [CD1].

Because the integer $d$ can be arbitarily large, the holomorphic mapping $A$ from Theorem 1 can have an arbitrarily large number of components. This fact has consequences for proper holomorphic mappings between balls in different dimensions. For example, in [CD1], Theorem 1 is used to prove the following. Given a holomorphic polynomial $q: \mathbf{C}^{\mathbf{n}} \rightarrow \mathbf{C}$ that doesn't vanish on the closed unit ball, there is an integer $N$ and a holomorphic polynomial mapping $p: \mathbf{C}^{\mathbf{n}} \rightarrow \mathbf{C}^{\mathbf{N}}$ such that $\frac{p}{q}$ is reduced to lowest terms, and defines a proper map between balls. The integer $N$ can be arbitarily large. This is in sharp contrast to the case when $n=1$, where the result is trivial, and we can take $N=1$ as well.

Next we give an application to symbols of differential operators. Let $D$ be a linear partial differential operator on real Euclidean space $\mathbf{R}^{\mathbf{2 n}}$ of even order $2 m$. Recall (See [F] for example) that the principal symbol, or characteristic form, $p(\xi)$ of $D$ governs whether it is elliptic. We suppose that the principal symbol has constant coefficients. Thus $p(\xi)=$ $\sum_{|\alpha|=2 m} c_{\alpha} \xi^{\alpha}$, and the operator is elliptic precisely when $p$ vanishes only at the origin. If we make the usual identification of $\mathbf{R}^{\mathbf{2 n}}$ with $\mathbf{C}^{\mathbf{n}}$, then we can express $D$ in terms of the operators $\frac{\partial}{\partial z_{j}}$ and $\frac{\partial}{\partial \bar{z}_{j}}$. Using multi-index notation we can then write the principal symbol as

$$
\sum_{|\alpha|+|\beta|=2 m} c_{\alpha \beta}\left(\frac{\partial}{\partial z}\right)^{\alpha}\left(\frac{\partial}{\partial \bar{z}}\right)^{\beta}=f\left(\frac{\partial}{\partial z}, \frac{\partial}{\partial \bar{z}}\right) .
$$

In general, $f$ is not bihomogeneous. There are simple simple necessary and sufficient condition for a real-valued polynomial $f(z, \bar{z})$ to be bihomogeneous of degree $2 \mathrm{~m}$. One is that it be both homogeneous of degree $2 m$ over $\mathbf{R}$ and invariant under replacing $z$ by $e^{i \theta} z$. (Here $e^{i \theta}$ is a scalar, not an $n$-tuple). Another is the existence of holomorphic polynomial mappings $A$ and $B$, each homogeneous of degree $m$, such that $f(z, \bar{z})=\|A(z)\|^{2}-\|B(z)\|^{2}$. An arbitrary real-valued polynomial $p$ can be written as the difference of squared norms of holomorphic polynomials, but the holomorphic polynomials will not be homogeneous of the same degree when $p$ fails to be bihomogeneous. See $[D]$ for uses of such a decomposition.

We say that the partial differential operator $D$ on $\mathbf{R}^{\mathbf{2 n}}$ is complex bihomogeneous if its principal symbol is a bihomogeneous polynomial. In this case we may apply Theorem 1 to obtain the following conclusion. We write $\triangle$ for the Laplace operator defined by $\sum \frac{\partial}{\partial z_{j}} \frac{\partial}{\partial \bar{z}_{j}}$.

Theorem 2. Let $D$ be a complex bihomogeneous linear partial differential operator. Suppose that $p$ is the absolute value of the principal symbol of $D$. Let $q_{d}$ be the absolute value of the principal symbol of $\triangle^{d} D$. The following are equivalent:

1) $D$ is elliptic (that is, $p(z, \bar{z})>0$ for $z \neq 0$ ).

2) There is an integer $d$ and a positive-definite matrix $\left(E_{\mu \nu}\right)$ so that $q_{d}$ satisfies 


$$
q_{d}\left(\frac{\partial}{\partial z}, \frac{\partial}{\partial \bar{z}}\right)=\sum_{|\mu|=m} \sum_{|\nu|=m} E_{\mu \nu}\left(\frac{\partial}{\partial z}\right)^{\mu}\left(\frac{\partial}{\partial \bar{z}}\right)^{\nu} .
$$

3) There is an integer $d$ so that $q_{d}$ is a squared norm of a holomorphic differential operator:

$$
q_{d}\left(\frac{\partial}{\partial z}, \frac{\partial}{\partial \bar{z}}\right)=\left\|\sum A_{\mu}\left(\frac{\partial}{\partial z}\right)^{\mu}\right\|^{2}=\sum_{i}\left|\sum A_{\mu i}\left(\frac{\partial}{\partial z}\right)^{\mu}\right|^{2} .
$$

We assume also that the indicated homogeneous polynomials span $V_{m+d}$.

4. There is an integer $d^{\prime}$ so that $q_{d^{\prime}}$ satisfies (23), and such that $\mathbf{V}(A)=\{0\}$.

Proof. The principal symbol of $\triangle^{d} D$, evaluated at $(z, \bar{z})$, is $q_{d}(z, \bar{z})=\|z\|^{2 d} p(z, \bar{z})$. The operator $D$ is elliptic precisely when $|p|$ is strictly positive away from the origin. Therefore, by Theorem 1, D is elliptic if and only there is $d$ so that $\|z\|^{2 d} p(z, \bar{z})$ satisfies any of the equivalent conditions of Proposition 1. Equation (10), applied when $r=1$, is equivalent to the positive-definiteness of $\left(E_{\mu \nu}\right)$. The strict holomorphic factorability there is equivalent to statement 3 here. Statement 3 obviously implies statement 4 , which in turn implies that $p(z, \bar{z})$ is positive away from the origin. Thus the four statements are equivalent.

Remark. This result extends to systems of PDE in a straightforward fashion.

\section{Reinterpretation of Theorem 1}

Next we reinterpret Theorem 1 in terms of pullbacks of the universal bundle over Grassman manifolds. See $[\mathrm{W}]$ for more details about the universal bundle. Let $\mathbf{G}_{p, N}$ denote the Grassman manifold of $p$ planes in complex $N$-space. When $p=1$ we have complex projective space, and we write as usual $\mathbf{P}^{N-1}$ for $\mathbf{G}_{1, N}$. Let $\mathbf{U}_{p, N}$ denote the universal bundle over $\mathbf{G}_{p, N}$. This bundle is sometimes known as the tautological bundle or the stupid bundle; a point in $\mathbf{U}_{p, N}$ is a pair $(S, \zeta)$ where $S$ is a $p$-dimensional subspace of $\mathbf{C}^{\mathbf{N}}$ and $\zeta \in S$.

We let $g_{0}$ denote the Euclidean metric on $\mathbf{U}_{p, N}$. In terms of a local frame $e$ of $\mathbf{U}_{p, N}$, we define $g_{0}(e)=e^{*} e$. Observe that if $T$ determines a change of frames by acting on the right, then

$$
g_{0}(e T)=(e T)^{*}(e T)=T^{*} e^{*} e T=T^{*} g_{0}(e) T .
$$

This is the correct transformation law, and hence $g_{0}$ defines a Hermitian metric on $\mathbf{U}_{p, N}$. We may consider the matrix representation of $g_{0}$, with respect to a local frame. We have $\left(g_{0}\right)_{i j}=\left\langle e_{j}, e_{i}\right\rangle$ where $\langle$,$\rangle denotes the usual Hermitian inner product on complex Euclidean$ space $\mathbf{C}^{\mathbf{N}}$, and the vectors $e_{i}$ for $i=1, \ldots, r$ are linearly independent. Note the interchange of indices. We see immediately that $g_{0}$ is of the form $A^{*} A$.

Let $L$ denote the universal line bundle $L=\mathbf{U}=\mathbf{U}_{1, n}$ over complex projective space $\mathbf{P}^{n-1}$. We consider also its $m$-th tensor power $\mathbf{U}^{m}$. Let $E$ denote the vector bundle over $\mathbf{P}^{n-1}$ equal to the direct sum of $r$ copies of $\mathbf{U}^{m}$. On $L$ we use the Euclidean metric, written $\|z\|^{2}$, and on $E$ we use the metric determined by a matrix of bihomogeneous polynomials $F(z, \bar{z})$ that is positive-definite for $z \neq 0$. Theorem 1 now admits the following restatement. 
Theorem 3. Suppose that $L$ and $E$ are the bundles over $\mathbf{P}^{n-1}$ as described in the previous paragraph, equipped with the given metrics. Then there are integers $N$ and $d$, so that the bundle $L^{d} \otimes E$ over $\mathbf{P}^{n-1}$, with metric determined by $\|z\|^{2 d} F(z, \bar{z})$, is the isometric pullback of the vector bundle $\mathbf{U}_{r, N}$, with the Euclidean metric, over the Grassmanian $\mathbf{G}_{r, N}$ via a holomorphic embedding.

The link to bihomogeneous polynomials arises because one can identify homogeneous polynomials of degree $m$ on $\mathbf{C}^{\mathbf{n}}$ with sections of the $m$-th power $H^{m}$ of the hyperplane bundle $H$ over $\mathbf{P}^{n-1}$. The bundle $H$ is dual to $\mathbf{U}$. A matrix of bihomogeneous polynomials determines a metric on the direct sum of $r$ copies of $\mathbf{U}$.

The general result in [CD3] considers certain base manifolds $M$, a line bundle $L$ and a vector bundle $E$ over $M$, and metrics $R$ and $F$ on them satisfying certain conditions. The conclusion again guarantees the existence of integers $N$ and $d$ so that $L^{d} \otimes E$, with metric $R^{d} F$, is the isometric pullback of $\mathbf{U}_{r, N}$, with the Euclidean metric, over the Grassmanian $\mathbf{G}_{r, N}$. The proof again relies on the Bergman kernel and facts about compact operators, but it is technically more difficult than the special case considered here.

\section{Remarks on classical factorization}

We briefly mention some of the results in $[\mathrm{Dj}]$ and $[\mathrm{RR}]$. Djokovic $[\mathrm{Dj}]$ considers for example an $r$ by $r$ positive semi-definite matrix $F(\lambda, \mu)$ whose entries are complex-valued homogeneous polynomials of degree $2 m$ in the pair of real variables $(\lambda, \mu)$. He proves that one can write $F(\lambda, \mu)=A(\lambda, \mu)^{*} A(\lambda, \mu)$ where the entries in $A$ are homogeneous polynomials of degree $m$. Two nice things about this result are that it is not required to multiply $F$ by powers of a scalar function, and $F$ is allowed to be semi-definite. On the other hand, the theorem holds only when the entries depend upon two real variables, the analogue of one complex variable. For us, making $A(z)$ depend holomorphically on $z$ in (4) requires that we work with bihomogneous polynomials. The only bihomogeneous polynomials in one complex variable are constants times $|z|^{2 m}$. Hence we could factor this scalar out of the matrix completely, and the analogue of the result in $[\mathrm{Dj}]$ becomes trivial in our setting. The idea of multiplying by powers of a scalar factor does not appear in $[\mathrm{Dj}]$.

The work in $[\mathrm{RR}]$ concerns functions from either $\mathbf{R}$ or the unit circle $S^{1}$ that take values in non-negative operators on a Hilbert space. The authors study many aspects of the factorization question in detail, including holomorphic extension to the upper half plane or to the unit disc. One of many results there is that if $P(x)$ is a non-negative operator on a Hilbert space, that is a polynomial of degree $2 m$ in the real variable $x$, then there is an operator $Q(x)$ such that $P(x)=Q(x)^{*} Q(x)$ and such that $Q$ is a polynomial of degree $m$ in $x$. Other results in $[\mathrm{RR}]$ are related to an application of Theorem 1 here from [CD1]. Suppose that $f(z, \bar{z})$ is an arbitrary polynomial that is positive on the unit sphere. Then there is a holomorphic polynomial mapping $g$ such that $f(z, \bar{z})=\|g(z)\|^{2}$ on the unit sphere. In $[R R]$ however positivity questions are considered only for functions depending on one variable.

\section{References}


[CD1] David W. Catlin and John P. D'Angelo, A stabilization theorem for Hermitian forms and applications to holomorphic mappings, Math Research Letters 3 (1996), 149-166.

[CD2] David W. Catlin and John P. D'Angelo, Positivity conditions for bihomogeneous polynomials, Math Research Letters 4 (1997), 1-13.

[CD3] David W. Catlin and John P. D'Angelo, Isometric embeddings of bundles, (in preparation).

[D] John P. D'Angelo, Several complex variables and the geometry of real hypersurfaces, CRC Press, Boca Raton, 1993.

[Dj] D. Z. Djokovic, Hermitian matrices over polynomial rings, J. Algebra 43 (1976), 359-374.

[F] Gerald B. Folland, Introduction to Partial Differential Equations, Princeton University Press, 1976.

[RR] M. Rosenblum and J. Rovnyak, The factorization problem for nonnegative operator valued functions, Bulletin A.M.S. 77 (1971), 287-318.

[Ru] Walter Rudin, Functional Analysis, McGraw-Hill, New York, 1973.

[W] Raymond O. Wells, Differential Analysis on Complex Manifolds, Prentice-Hall, Englewood Cliffs, New Jersey, 1973. 